\title{
Isolated right atrial appendage thrombus in transoesophageal echocardiogram before invasive intervention
}

\author{
Juan Asensio-Nogueira, Luis F. Iglesias-Alonso \\ Department of Cardiology, Universitary Hospital of Burgos, Spain
}

A 79-year-old male patient was scheduled for percutaneous left atrial appendage (LAA) closure. He had history of atrial fibrillation and had been admitted to Neurology 1 month prior for left thalamic hemorrhage. After anticoagulation was reversed with prothrombin complex and $\mathrm{K}$ vitamin and acenocumarol was suspended, he presented with new neurological symptoms, and cerebral magnetic resonance showed several ischemic infarctions in the right hemisphere. This prompted the current intervention, upon performance of a transoesophageal echocardiogram (TEE).

The imaging test showed spontaneous echocardiographic contrast in left atrium and LAA without thrombus (Fig. 1A); it also showed an organized thrombus in right atrial appendage (RAA) (white arrows in Fig. 1B, black arrow in Fig. 1C).

Intervention was suspended. Due to a history of cerebral hemorrhage under treatment with acenocumarol, it was decided to initiate anticoagulation treatment with apixaban, which has a better security profile than acenocumarol. TEE performed after 4 weeks under anticoagulant treatment showed complete disappearance of the thrombus (Fig. 1D, E).

Right atrial appendage thrombi are much less frequent that than their left-sided counterparts, probably due to differences in appendage anatomy and function. Cases of isolated RAA thrombus are extremely rare. Atrial fibrillation, right ventricular dysfunction and pulmonary hypertension are factors associated with the development of thrombi in this location.

Although thrombi in the RAA are rare, this case underlines the importance of an asessment of this structure in patients who undergo percutaneous interventions, particularly if associated factors are present.

\section{Acknowledgments}

Thanks to Virginia Pascual-Tejerina and Nuria Sánchez-Mata for acquiring the echocardiography images.

Conflict of interest: None declared

Address for correspondence: Dr. Juan Asensio-Nogueira, Department of Cardiology, Universitary Hospital of Burgos, Avenida Islas Baleares, 3, 09006 Burgos, Spain, tel: +34 628323 176, e-mail: jasensionogueira@gmail.com 


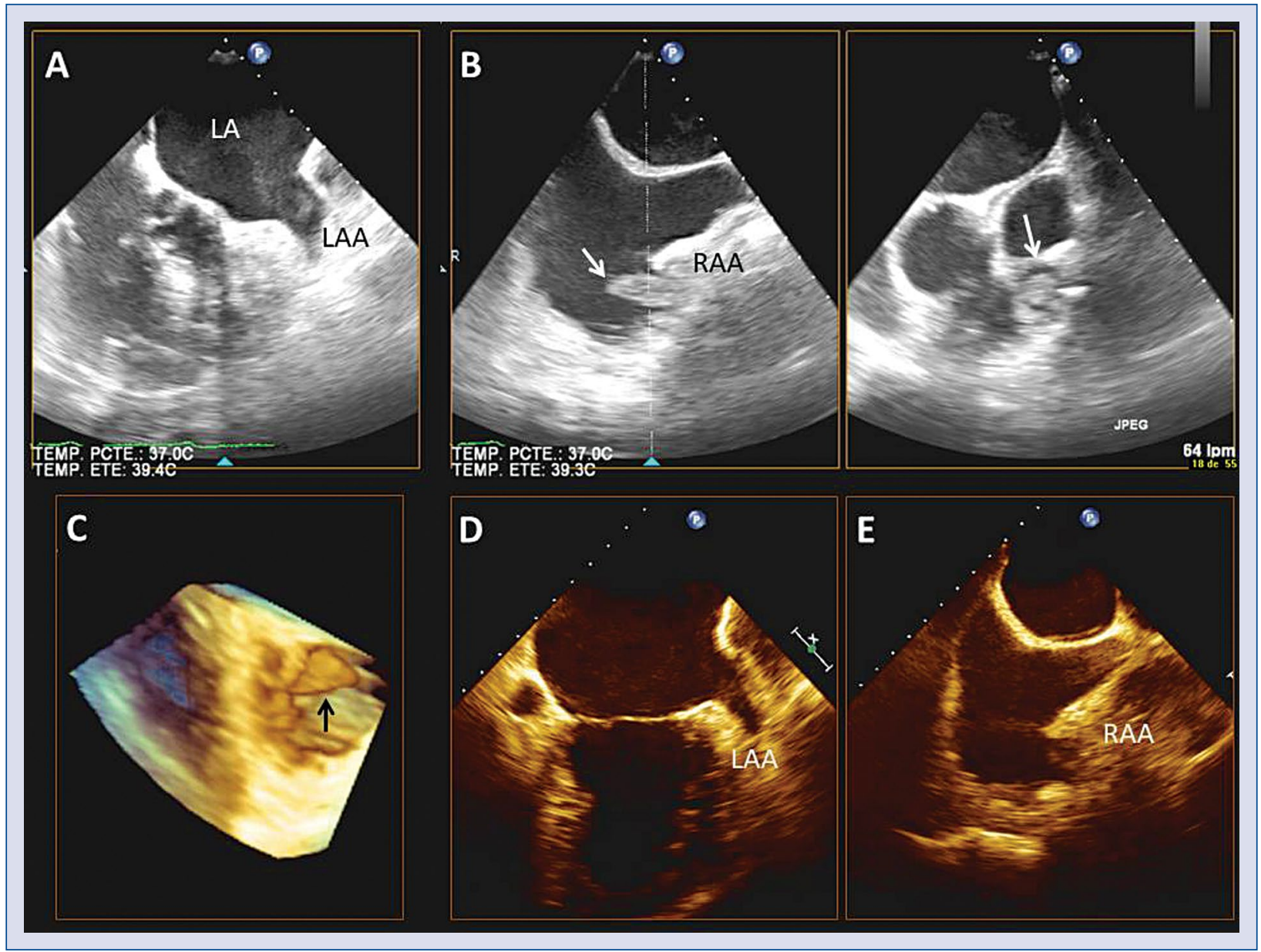

Figure 1. Transoesophageal echocardiography (TEE); A. Modified short axis view showing spontaneous echocardiographic contrast in left atrium (LA) and left atrial appendage (LAA); B. Upper transoesophageal biplane views showing the right atrium. An organized thrombus can be observed in the right atrial appendage (RAA; white arrows); C. Three-dimensional image of the thrombus (black arrow); D, E. Control TEE showing complete disappearance of the thrombus. 